\title{
Phase properties of elastic waves in systems constituted of adsorbed diatomic molecules on the (001) surface of a simple cubic crystal
}

\section{P. A. Deymier, and K. Runge}

Citation: Journal of Applied Physics 123, 125106 (2018); doi: 10.1063/1.5007049

View online: https://doi.org/10.1063/1.5007049

View Table of Contents: http://aip.scitation.org/toc/jap/123/12

Published by the American Institute of Physics

\section{Articles you may be interested in}

Improvement of contact grating device for efficient terahertz wave generation using bi-angular filter Journal of Applied Physics 123, 123104 (2018); 10.1063/1.5025194

Heterodyne transverse velocimetry for pressure-shear plate impact experiments Journal of Applied Physics 123, 125902 (2018); 10.1063/1.5023007

Incommensurately modulated phase and charge ordering transition in nanocrystalline $\mathrm{Nd}_{0.5} \mathrm{Sr}_{0.5} \mathrm{MnO}_{3}$ perovskite

Journal of Applied Physics 123, 124301 (2018); 10.1063/1.5007199

Manipulating acoustic wave reflection by a nonlinear elastic metasurface

Journal of Applied Physics 123, 124901 (2018); 10.1063/1.5015952

Optical properties of InGaN thin films in the entire composition range Journal of Applied Physics 123, 125101 (2018); 10.1063/1.5020988

Investigation of acoustic metasurfaces with constituent material properties considered Journal of Applied Physics 123, 124905 (2018); 10.1063/1.5007863

\section{PHYSICS TODAY}

MANAGER'S GUIDE WHITEPAPERS

\section{READ NOW}

PRESENTED BY

Accelerate R\&D with
Multiphysics Simulation 


\title{
Phase properties of elastic waves in systems constituted of adsorbed diatomic molecules on the (001) surface of a simple cubic crystal
}

\author{
P. A. Deymier ${ }^{\text {a) }}$ and K. Runge ${ }^{\text {a) }}$ \\ Department of Materials Science and Engineering, University of Arizona, Tucson, Arizona 85721, USA
}

(Received 29 September 2017; accepted 11 March 2018; published online 28 March 2018)

\begin{abstract}
A Green's function-based numerical method is developed to calculate the phase of scattered elastic waves in a harmonic model of diatomic molecules adsorbed on the (001) surface of a simple cubic crystal. The phase properties of scattered waves depend on the configuration of the molecules. The configurations of adsorbed molecules on the crystal surface such as parallel chain-like arrays coupled via kinks are used to demonstrate not only linear but also non-linear dependency of the phase on the number of kinks along the chains. Non-linear behavior arises for scattered waves with frequencies in the vicinity of a diatomic molecule resonance. In the non-linear regime, the variation in phase with the number of kinks is formulated mathematically as unitary matrix operations leading to an analogy between phase-based elastic unitary operations and quantum gates. The advantage of elastic based unitary operations is that they are easily realizable physically and measurable. Published by AIP Publishing. https://doi.org/10.1063/1.5007049
\end{abstract}

\section{INTRODUCTION}

Our past and current understanding of sound and elastic waves has been nourished essentially by the paradigm of the plane wave and its periodic counterpart (the Bloch wave) in periodic media. This paradigm relies on the four canonical characteristics of waves: frequency $(\omega)$; wave vector $(\mathrm{k})$; amplitude (A); and phase $(\phi)$. Over the past two decades, the fields of phononic crystals and acoustic metamaterials have developed in which researchers manipulate the spectral and refractive properties of phonons and sound waves through their host material by exploiting $\omega$ and $\mathrm{k} .{ }^{1}$ The spectral properties of elastic waves include phenomena such as the formation of stop bands in the transmission spectrum due to Bragg-like scattering or resonant processes, as well as the capacity to achieve narrow band spectral filtering by introducing defects in the material's structure. Negative refraction, zero-angle refraction, and other unusual refractive properties utilize the complete characteristics of the dispersion relations of the elastic waves, $\omega(\mathrm{k})$, over both frequency and wave number domains. More recently, renewed attention has been paid to the amplitude and the phase characteristics of the elastic waves. ${ }^{2}$ For instance, when sound waves propagate in media under symmetry breaking conditions, they may exhibit amplitudes $A(k)=A_{0} e^{i \eta(k)}$ that acquire a geometric phase $\eta$ leading to non-conventional topology. ${ }^{2}$ In Ref. 2, the notion of the geometric phase was related to that of elastic Green's functions. In particular, it was shown that for Hermitian operators like those of elastic dynamical systems, the notion of the Berry connection ${ }^{3}$ is proportional to the imaginary part of its Green's function. This notion was illustrated in the case of the one-dimensional harmonic crystal with a finite length harmonic side branch acting as a scatterer. In that case, the scattering phase (i.e., Friedel phase ${ }^{4}$ )

\footnotetext{
${ }^{a)}$ Electronic addresses: deymier@email.arizona.edu and krunge@email. arizona.edu
}

and the phase of the transmission amplitude for the resonating side-branch were related to the geometric phase.

In this paper, we consider more complex elastic models where diatomic molecules are adsorbed on the twodimensional (001) cleaved surface of a cubic harmonic crystal. In Sec. II of this paper, we develop a model and methods that enable the calculation of the scattering phase and density of states of elastic waves upon adsorption of diatomic molecules on the surface of the cubic lattice. The method is based on the Interface Response Theory (IRT) $)^{5,6}$ which permits the calculation of the Green's function of elastically coupled components (adsorbed molecules on the surface) in terms of the Green's function of the individual components (uncoupled molecules and free surface). In Sec. III, we consider a number of configurations of adsorbed diatomic molecules on the surface of the crystal. More specifically, we pay attention to chain-like configurations of adsorbed diatomic molecules separated by first, second, third, and fourth nearest neighbor distances on the surface. The configurations are composed of diatomic molecules forming chain-like segments. We are particularly interested in a configuration composed of parallel chainlike segments with ends at fixed position with various types of kinks that can be formed between the chains. We focus on the effect of the number and nature of kinks on the scattered phase and density of states. More specifically, we show that in some spectral regime, the phase of chains with kinks relative to the phase of chains without kinks scales linearly with the number of kinks, that is, the phase effect of the kinks is additive so that they do not interact with each other. Furthermore, at frequencies near the resonant frequency of the diatomic molecule, we observe deviations from the linear behavior suggesting some more complex behavior due to interactions between kinks. The effect of the creation of kinks is modeled as unitary matrix transformations on the states of the elastic scattered waves of the systems. In Sec. IV, we draw an analogy between the 
phase-based unitary transformations that result from the creation of kinks in the configurations of adsorbed molecules. Finally, we draw conclusions in Sec. V, and in particular, on the possibility of using the scattered phase to store information and the manipulation of the configurations of diatomic molecules to process information.

\section{MODELS AND METHODS}

In this section, we develop the model of a cleaved semiinfinite simple elastic harmonic cubic crystal with a surface that can adsorb harmonic diatomic molecules. The masses in the cubic crystals are all identical and connected via harmonic springs along the edges of the simple cubic lattice. The diatomic molecules are constituted of two masses connect via a single harmonic spring. For the sake of mathematical tractability, the dynamic equations of the elastic system are limited to displacements of masses in one single direction. The direction of the displacement is unspecified and could be representing either transverse or longitudinal polarization. IRT ${ }^{5,6}$ enables us to calculate the elastic Green's function (more specifically the diffusion matrix) of the surface with adsorbed molecules. The variation in phase and density of states, relative to a system composed of the surface and uncoupled molecules, is calculated from the determinant of the diffusion matrix.

\section{A. 1-D molecule adsorbed on the (100) surface of a simple cubic crystal}

The calculation of the Green's function of a system composed of a semi-infinite simple cubic crystal (cleaved along the (001) face) and a finite 1-D monatomic coupled to a surface atom via a spring with constant, $\beta_{I}$, begins with the block matrix describing the Green's function of the uncoupled system $\left(\beta_{I}=0\right)$

$$
\overleftrightarrow{G}_{S}=\left(\begin{array}{cc}
\overleftrightarrow{g}_{S 1} & \stackrel{\leftrightarrow}{0} \\
\stackrel{\leftrightarrow}{0} & \overleftrightarrow{\mathrm{g}}_{S 2}
\end{array}\right)
$$

where $\stackrel{\leftrightarrow}{g}_{S 1}$ is the Green's function of the semi-infinite crystal and where $\overleftrightarrow{g}_{s 2}$ is the Green's function of the diatomic molecule. $\stackrel{\leftrightarrow}{S}_{S 1}$, therefore, describes elastic waves with any polarization in the three-dimensional semi-infinite space of the cleaved crystal. For the sake of simplicity, we are choosing the masses and spring constants of the crystal and molecules to be identical, $m$ and $\beta$. The spacing between masses along the molecule is $a$. The Green's function of a finite harmonic polymer of length $L$ (for $n, n^{\prime} \epsilon[1, L]$ ), with coordinates along the polymer expressed as integer multiples of that spacing: $x=n a,{ }^{2}$ is given by

$$
\begin{aligned}
g_{S 2}\left(n, n^{\prime}\right)= & \frac{m^{\prime}}{\beta^{\prime}}\left[\frac{t^{\left|n-n^{\prime}\right|+1}+t^{n+n^{\prime}}}{t^{2}-1}+\frac{t^{2 L+1}}{\left(t^{2}-1\right)\left(1-t^{2 L}\right)}\right. \\
& \left.\times\left(t^{n^{\prime}-n}+t^{n-n^{\prime}}+t^{1-n-n^{\prime}}+t^{n+n^{\prime}-1}\right)\right],
\end{aligned}
$$

where

$$
t= \begin{cases}\xi-\left(\xi^{2}-1\right)^{1 / 2} & \text { if } \xi>1 \\ \xi+\left(\xi^{2}-1\right)^{1 / 2} & \text { if } \xi<-1 \\ \xi+i\left(1-\xi^{2}\right)^{1 / 2} & \text { if }-1 \leq \xi \leq 1\end{cases}
$$

with

$$
\xi=1-\frac{m \omega^{2}}{2 \beta} .
$$

The frequency $\omega \in\left[0, \omega_{0}\right]$ with $\omega_{0}=2 \sqrt{\frac{\beta}{m}}$. A diatomic molecule is a polymer with $L=2$.

The Green's function of the semi-infinite cubic crystal possesses translational periodicity in the plane of the (001) surface and is written as a two-dimensional Fourier transform

$$
\begin{aligned}
\overleftrightarrow{g}_{S 1}\left(\omega, x_{1}, x_{2}, x_{3}\right)= & \frac{1}{(2 \pi)^{2}} \int_{-\frac{\pi}{a}}^{\frac{\pi}{a}} d k_{1} \int_{-\frac{\pi}{a}}^{\frac{\pi}{a}} d k_{2} e^{i\left(k_{1} x_{1}+k_{2} x_{2}\right)} \\
& \times \overleftrightarrow{\mathrm{g}}_{S 1}\left(\omega, k_{1}, k_{2}, x_{3}\right) .
\end{aligned}
$$

Here, $\left(x_{1}, x_{2}\right)$ is a site on the (001) surface of the semiinfinite crystal. The surface is located at $x_{3}=0$. The lattice parameter of the simple cubic crystal is also taken as $a$. The Fourier transform of the Green's function $\stackrel{\leftrightarrow}{g}_{S 1}$ is given by ${ }^{7}$

$$
\overleftrightarrow{g}_{S 1}\left(\omega, k_{1}, k_{2}, x_{3}\right)=\frac{m}{\beta} \frac{t^{\left|n_{3}-n_{3}^{\prime}\right|+1}+t^{2-\left(n_{3}+n_{3}^{\prime}\right)}}{t^{2}-1}
$$

with $n_{3}^{(\prime)}=\frac{x_{3}^{(\prime)}}{a}$.

We note that $g_{S 1}\left(\omega, x_{1}, x_{2}, x_{3}=0\right)$ is calculated as the two-dimensional Fourier transform [Eq. (5) of Eq. (6)]. Equation (6) requires the calculation of $t$ using Eq. (3). For the Fourier transform, we use the dispersion relation for a simple cubic crystal: $\omega^{2}=\frac{2 \beta}{m}\left(3-\cos k_{1} a-\cos k_{2} a-\cos k_{2} a\right)$ to define

$$
\xi=3-\cos k_{1} a-\cos k_{2} a-\frac{m \omega^{2}}{2 \beta} .
$$

If we define a position on the surface of the crystal: $p_{i}=\left(x_{1}^{(i)}, x_{2}^{(i)}, 0\right)$, then we only calculate for every frequency: $g_{S 1}\left(p_{i} p_{j}\right)=g_{S 1}\left[p_{i}-p_{j}=\left(x_{1}^{(j)}-x_{1}^{(i)}, x_{2}^{(j)}-x_{2}^{(i)}, 0\right)\right]$ for $\left(x_{1}^{(j)}\right.$ $\left.-x_{1}^{(i)}, x_{2}^{(j)}-x_{2}^{(i)}, 0\right)=\{(0,0,0),(0,1 a, 0),(0,2 a, 0),(1 a, 0,0)$, $(2 a, 0,0),(1 a, 1 a, 0),(1 a, 2 a, 0),(2 a, 1 a, 0)\}$. All other $g_{S 1}$ are neglected in this paper. Hence, we consider a cut off of $\left|p_{i}-p_{j}\right|=\sqrt{5} a$ for the Green's function $g_{S 1}\left(p_{i} p_{j}\right)$. We denote by $g_{S 1}\left(\left|p_{i}-p_{j}\right|=0\right)=g_{00}, g_{S 1}\left(\left|p_{i}-p_{j}\right|=1 a\right)=g_{01}$, $g_{S 1}\left(\left|p_{i}-p_{j}\right|=2 a\right)=g_{02}, g_{S 1}\left(\left|p_{i}-p_{j}\right|=\sqrt{2} a\right)=g_{11}$, and $g_{S 1}\left(\mid p_{i}\right.$ $\left.-p_{j} \mid=\sqrt{5} a\right)=g_{12}$. The numerical functions $g_{S 1}\left(p_{i} p_{j}, \omega\right)$ are replaced by fits to functions of frequency, $\omega$. These fits are given in the Appendix.

Following the IRT, we define a coupling operator that enables us to couple a site 1 of a diatomic molecule to a site $X$ [effectively $X=\left(x_{1}, x_{2}, x_{3}=0\right)$ ] on the surface of the cubic crystal 


$$
\overleftrightarrow{V}_{I}=\left(\begin{array}{cc}
V_{I}(X, X) & V_{I}(X, 1) \\
V_{I}(1, X) & V_{I}(1,1)
\end{array}\right)=\left(\begin{array}{cc}
\frac{-\beta_{I}}{m} & \frac{\beta_{I}}{m} \\
\frac{\beta_{I}}{m} & \frac{-\beta_{I}}{m}
\end{array}\right) .
$$

For the sake of simplicity, we will take: $\beta_{I}=\beta$.

IRT introduces the surface operator expressed in the space $\mathrm{M}$ of coupled interface sites

$$
\vec{A}_{0}(M M)=\left(\begin{array}{c}
A(X, X) \\
A(X, 1) \\
A(1, X) \\
A(1,1)
\end{array}\right)=\left(\begin{array}{c}
V_{I}(X, X) g_{S 1}(X, X) \\
V_{I}(X, 1) g_{S 2}(1,1) \\
V_{I}(1, X) g_{S 1}(1, X) \\
V_{I}(1,1) g_{S 2}(1,1)
\end{array}\right) .
$$

The diffusion matrix takes then the form of a $2 \times 2$ matrix in the space of the interface sites, $M$

$$
\begin{aligned}
\overleftrightarrow{\Delta}(M M) & =\left(\begin{array}{cc}
1+A(X, X) & A(X, 1) \\
A(1, X) & 1+A(1,1)
\end{array}\right) \\
& =\left(\begin{array}{cc}
1+V_{I}(X, X) g_{S 1}(X, X) & V_{I}(X, 1) g_{S 2}(1,1) \\
V_{I}(1, X) g_{S 1}(1, X) & 1+V_{I}(1,1) g_{S 2}(1,1)
\end{array}\right) .
\end{aligned}
$$

The phase difference (normalized to $\pi$ ) of elastic modes in the space $\mathrm{M}$ between the coupled system and the uncoupled system is then obtained from the relation

$$
\eta(\omega)=-\frac{1}{\pi} \operatorname{Im}[\ln (\operatorname{det} \overleftrightarrow{\Delta}(M M))] .
$$

This is effectively the phase accumulated by the wave scattered by the adsorbed diatomic molecules.

The variation in density of states due to the coupling is then obtained from the relation

$$
\vec{G}_{S}(M M)=\left(\begin{array}{ccccccccc}
g_{S 1}\left(p_{1} p_{1}\right) & 0 & g_{S 1}\left(p_{1} p_{2}\right) & 0 & g_{S 1}\left(p_{1} p_{3}\right) & 0 & \ldots & g_{S 1}\left(p_{1} p_{N_{c}}\right) & 0 \\
0 & g_{S 2}(11) & 0 & 0 & 0 & 0 & \ldots & 0 & 0 \\
g_{S 1}\left(p_{2} p_{1}\right) & 0 & g_{S 1}\left(p_{2} p_{2}\right) & 0 & g_{S 1}\left(p_{2} p_{3}\right) & 0 & \ldots & g_{S 1}\left(p_{2} p_{N_{c}}\right) & 0 \\
0 & 0 & 0 & g_{S 2}\left(1^{\prime} 1^{\prime}\right) & 0 & 0 & \ldots & 0 & 0 \\
g_{S 1}\left(p_{3} p_{1}\right) & 0 & g_{S 1}\left(p_{3} p_{2}\right) & 0 & g_{S 1}\left(p_{3} p_{3}\right) & 0 & \ldots & g_{S 1}\left(p_{3} p_{N_{c}}\right) & 0 \\
0 & 0 & 0 & 0 & 0 & g_{S 2}\left(1^{(2)} 1^{(2)}\right) & \ldots & 0 & 0 \\
\vdots & \vdots & \vdots & \vdots & \vdots & \vdots & \vdots & \vdots & \vdots \\
g_{S 1}\left(p_{N_{c}} p_{1}\right) & 0 & g_{S 1}\left(p_{N_{c}} p_{2}\right) & 0 & g_{S 1}\left(p_{N_{c}} p_{3}\right) & 0 & \ldots & g_{S 1}\left(p_{N_{c}} p_{N_{c}}\right) & 0 \\
0 & 0 & 0 & 0 & 0 & 0 & \ldots & 0 & g_{S 2}\left(1^{\left(N_{c}-1\right)} 1^{\left(N_{c}-1\right)}\right)
\end{array}\right) .
$$

$$
\Delta n(\omega)=\frac{d \eta(\omega)}{d\left(\omega^{2}\right)} .
$$

$\Delta n$ is the variation of the phonon density of states between the adsorbed system and the reference system (i.e., crystal with uncoupled diatomic molecules).

\section{B. Multiple diatomic molecules adsorbed on the surface of a cubic crystal}

We now consider $N_{c}$ identical diatomic molecules grafted on the (001) surface of the simple cubic crystal. The space $M$ for the system is now defined as

$$
\begin{aligned}
M & =\left\{p_{1}=(0,0,0), 1, p_{2}=\left(x_{1}^{\prime}, x_{2}^{\prime}, 0\right), 1^{\prime}, p_{3}\right. \\
& \left.=\left(x_{1}^{\prime \prime}, x_{2}^{\prime \prime}, 0\right), 1^{\prime \prime}, \ldots, p_{N_{c}}=\left(x_{1}^{\left(N_{c}-1\right)}, x_{2}^{\left(N_{c}-1\right)}, 0\right), 1^{\left(N_{c}-1\right)}\right\} .
\end{aligned}
$$

We have located the first finite chain at the origin on the crystal surface. In this case, the coupling operator is a $2 N_{c}$ $\times 2 N_{c}$ matrix of the form

$$
\vec{V}_{I}=\frac{\beta_{I}}{m}\left(\begin{array}{ccccccc}
-1 & 1 & 0 & 0 & \ldots & 0 & 0 \\
1 & -1 & 0 & 0 & \ldots & 0 & 0 \\
0 & 0 & -1 & 1 & \ldots & 0 & 0 \\
0 & 0 & 1 & -1 & \ldots & 0 & 0 \\
\vdots & \vdots & \vdots & \vdots & \ldots & \vdots & \vdots \\
0 & 0 & 0 & 0 & 0 & -1 & 1 \\
0 & 0 & 0 & 0 & 0 & 1 & -1
\end{array}\right) .
$$

To calculate $\overleftrightarrow{\Delta}(M M)=\overleftrightarrow{I}(M M)+\overleftrightarrow{V}_{I}(M M) \vec{G}_{S}(M M)$, one needs the Green's function of the uncoupled system, $\vec{G}_{S}(M M)$, which takes the form
In this matrix, the odd entries (rows or columns) correspond to locations on the surface of the crystal and the even 
The diffusion matrix in the space $M$ is

$$
\overleftrightarrow{\Delta}(M M)=\overleftrightarrow{I}+\overleftrightarrow{A}(M, M)=\overleftrightarrow{I}+\overleftrightarrow{V}_{I} \overleftrightarrow{G}_{S}(M M) .
$$

The phase difference is again given by Eq. (11).

\section{RESULTS AND DISCUSSION}

In this section, we analyze several configurations of diatomic molecules adsorbed on the (001) surface of the cubic crystal. The analysis focuses on density of states and more importantly variation in the scattered phase of elastic modes relative to uncoupled reference systems. After the characterization of a single adsorbed molecule, we focus on configurations of many adsorbed molecules that take the form of parallel linear chains and parallel chains with kinks along their length.

\section{A. One molecule adsorbed on the (001) surface of a cubic crystal}

Here, we consider one diatomic absorbed on the surface of the cubic crystal. As stated in Sec. II, all masses are the same and take the value 1 . All spring constant are identical and also take the value 1 . This system is illustrated in Fig. 1.

The variation in density of states exhibits two peaks corresponding to resonances between the crystal and the molecule. The resonances occur at frequencies 0.5570 and 1.5630 . The phase shows an inconsequential $2 \pi$ jump, two continuous $\pi$ phase changes, and one discontinuous $\pi$ phase change.
The continuous variations in phase, which are associated with the Friedel phase, ${ }^{4}$ correspond to the resonances. The derivative of the Friedel phase is directly related to the variation in the density of states. The Friedel phase continuously accumulates $\pi$ each time the frequency crosses a bound state in the diatomic molecule. The discontinuous phase change is the phase change associated with the zeros of the transmitted amplitude of the scattered wave. As frequency increases, each time the scattered amplitude passes through a zero, its real part becomes zero and its imaginary part changes sign. The ratio of the imaginary part to the real part diverges with a sign change across the zero of amplitude. The phase of the diffused wave then exhibits a phase slip of $\pi$. This behavior has been observed experimentally and discussed theoretically in systems of lower dimensionality for other excitations than phonons, namely electronic excitations. ${ }^{8-10}$

If one considers a random distribution of $N_{c}$ diatomic molecules on the surface with intermolecular spacing $\left|p_{i}-p_{j}\right|$ in excess of $\sqrt{5} a$, one expects no "interactions" between the molecules through the substrate. We use quotation marks to define "interaction" of diatomic molecules through the substrate. The variation in density of states for that system and subsequently its phase will be essentially the same (to a scaling factor dependent on the number of molecules) as that of Fig. 2. In that case, the Green's function of the uncoupled system (and subsequently the diffusion matrix), will take a simpler form

$$
\begin{aligned}
\overleftrightarrow{G}_{s}(M M) & =\left(\begin{array}{ccccccccc}
g_{S 1}\left(p_{1} p_{1}\right) & 0 & 0 & 0 & 0 & 0 & \ldots & 0 & 0 \\
0 & g_{S 2}(11) & 0 & 0 & 0 & 0 & \ldots & 0 & 0 \\
0 & 0 & g_{S 1}\left(p_{2} p_{2}\right) & 0 & 0 & 0 & \ldots & 0 & 0 \\
0 & 0 & 0 & g_{S 2}\left(1^{\prime} 1^{\prime}\right) & 0 & 0 & \ldots & 0 & 0 \\
0 & 0 & 0 & 0 & g_{S 1}\left(p_{3} p_{3}\right) & 0 & \ldots & 0 & 0 \\
0 & 0 & 0 & 0 & 0 & g_{S 2}\left(1^{(2)} 1^{(2)}\right) & \ldots & 0 & 0 \\
\vdots & \vdots & \vdots & \vdots & \vdots & \vdots & \vdots & \vdots & \vdots \\
0 & 0 & 0 & 0 & 0 & 0 & \ldots & g_{S 1}\left(p_{N_{c}} p_{N_{c}}\right) & 0 \\
0 & 0 & 0 & 0 & 0 & 0 & \ldots & 0 & g_{S 2}\left(1^{\left(N_{c}-1\right)} 1^{\left(N_{c}-1\right)}\right)
\end{array}\right) \\
& =\left(\begin{array}{cc}
g_{S 1}\left(p_{1} p_{1}\right) & 0 \\
0 & g_{S 2}(11)
\end{array}\right) \otimes\left(\begin{array}{cc}
1 & 0 \\
0 & 1
\end{array}\right) \otimes \ldots \otimes\left(\begin{array}{cc}
1 & 0 \\
0 & 1
\end{array}\right) .
\end{aligned}
$$

The Hilbert space of the system of $N_{c}$ diatomic molecules randomly distributed on sites on the surface is the tensor product of the Hilbert space on each site. Relative to such a tensor product space, the elastic state of the system can be also written as the tensor product of the state of each adsorbed molecule. This is the general definition of a local system. However, if the diatomic molecules are in close proximity (with nearest neighbor, second nearest neighbor, etc. separations distances on the surface lattice) and forming ordered patterns with "interactions" defined by the terms of the form: $g_{S 1}\left(p_{i} p_{j}\right), i \neq j$, the diffusion matrix may not be factorizable into a tensor product of individual adsorbed diatomic molecules. The elastic states' ordered patterns will be therefore non-separable relative to the states of individual adsorbed diatomic molecules. This reflects the collective nature of vibrational modes of configurations of adsorbed diatomic molecules that interact through the substrate. Systems composed of chain-like configurations of adsorbed molecules are discussed in Sec. III B. 


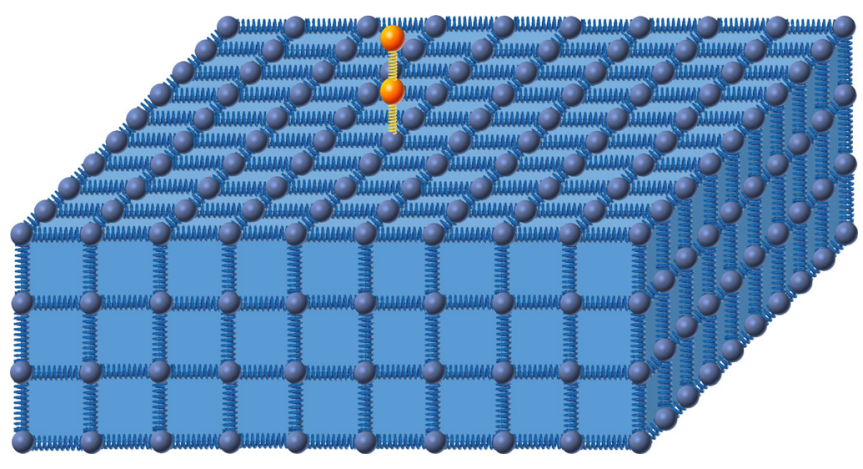

FIG. 1. Schematic illustration of a diatomic molecule adsorbed on the surface [top face (001)] of a semi-infinite simple cubic crystal. The crystal extends to infinity in all other directions.

\section{B. Chains of diatomic molecules adsorbed on the surface of a cubic crystal along the (100) direction}

In Fig. 3, we consider five configurations of adsorbed diatomic molecules on the crystal surface. The first configuration consists of two parallel arrays of molecules. The molecules are arranged along the edge of the unit cell of the twodimensional lattice of the surface. Each array contains 30 molecules. The separation distance between the two chains is $3 a$ along the vertical direction ensuring that any two diatomic molecules located on different chains are not
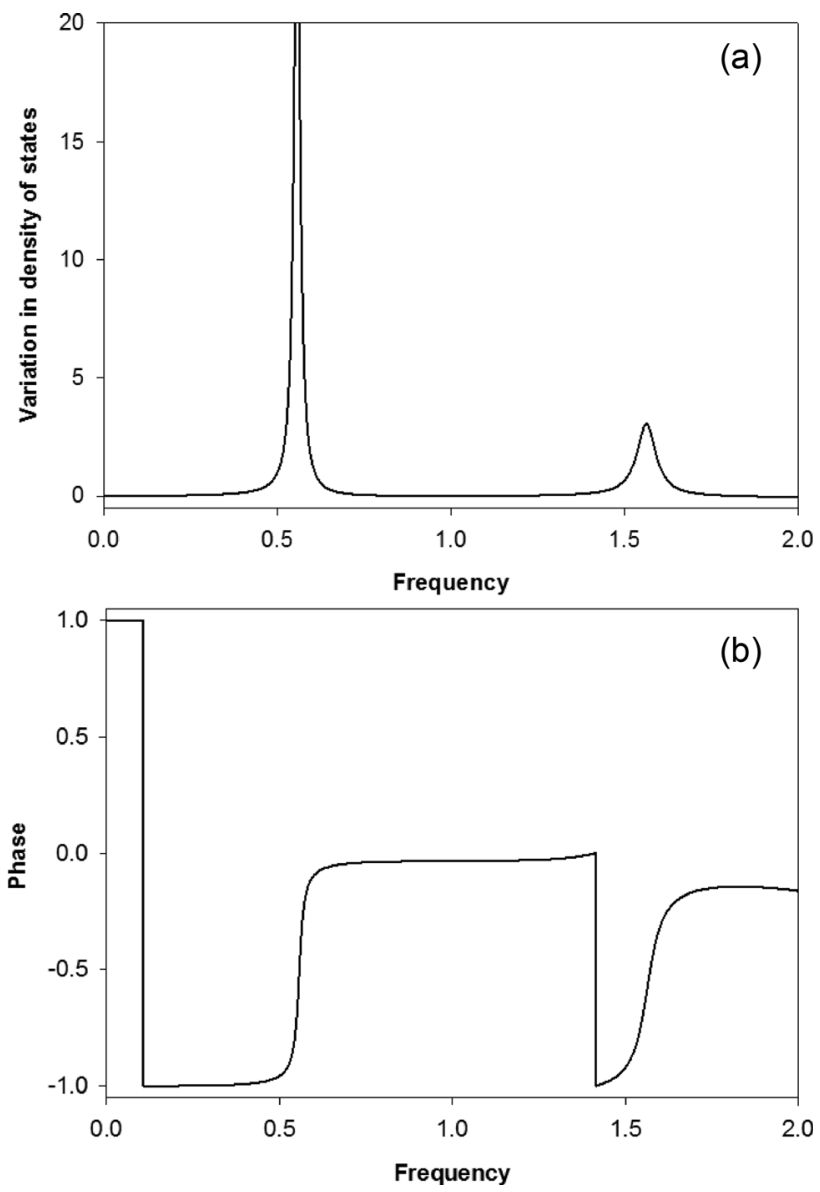

FIG. 2. (a) Variation in density of states, $\Delta n(\omega)$, of the system composed of a diatomic molecule adsorbed on the (001) surface of a simple cubic crystal. (b) Phase, $\eta(\omega)$, for the same system. The frequency range, $\omega \in[0,2]$ corresponds to the band of the 1-D monatomic harmonic crystal with $m=\beta=1$. (a)

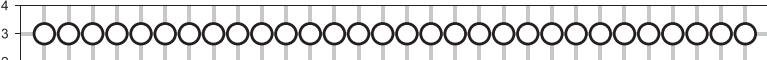

(b)

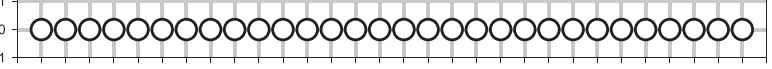

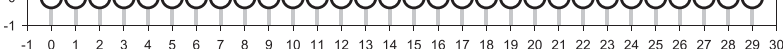
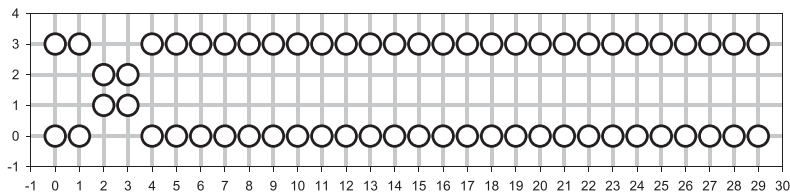

(c)

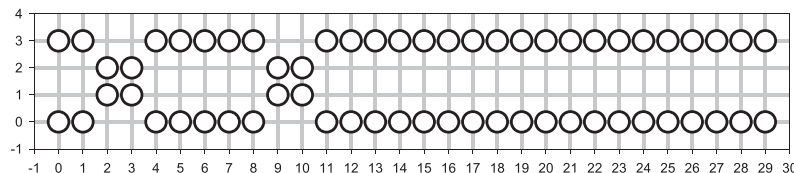

(d)
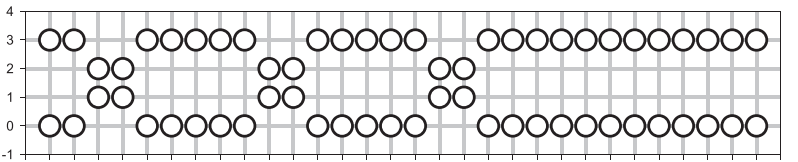

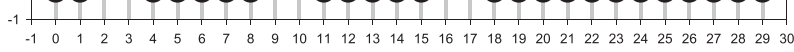

(e)

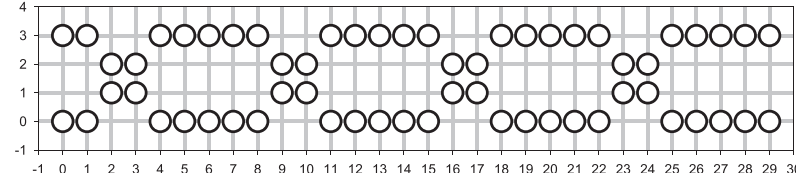

FIG. 3. Top view of the five configurations of $N_{c}=60$ diatomic molecules adsorbed on the (001) surface of the simple cubic crystal. The surface is illustrated as a square lattice. All diatomic molecules are identical. (a) Two linear parallel arrays of diatomic molecules, the separation distance is such that the two chains cannot "interact" through the substrate. (b), (c), (d), and (e) parallel configurations of two chains subjected to one, two, three, and four kinks, respectively.

coupled through a $g_{S 1}\left(p_{i} p_{j}\right)$ term in the matrix of Eq. (15). The two chains are not "interacting" through the substrate. In Figs. 3(b)-3(e), we have created a successive number of kinks occurring periodically along the chains while the position of the ends of the chains remains fixed.

The kinks are realized by displacing two diatomic molecules in each chain up and down by $a$ from the positions in the two parallel chain configuration [Fig. 3(a)]. The constraints imposed on these configurations are that the number of adsorbed molecules is constant and equal to $N_{c}=60$, and that the ends of the chains are always kept at the same coordinates on the surface. The variation in density of states for the parallel chains configurations [Fig. 3(a)] is reported in Fig. 4. The variation in density of states exhibits a wide region of large negative values above the first resonance of an individual adsorbed molecule (frequency 0.5570). This is characteristic of the formation of a resonant "gap" above that frequency. This is not a true gap as the number of molecules in each chain is finite. But the finite system reflects the behavior of its infinite counterpart in the form of a finite depression in the density of states. This "gap" results from the hybridization of the lower frequency mode of diatomic molecules and the substrate modes. Above and below this gap, the elastic modes are dispersive and the frequency now depends on a parameter that can be defined as a "wave number" along the direction of the chains. The dispersive modes cannot be expressed as a tensor product of states of individual adsorbed molecules. Indeed, the modes of $N_{c}=60$ randomly well-separated diatomic molecules would have the 


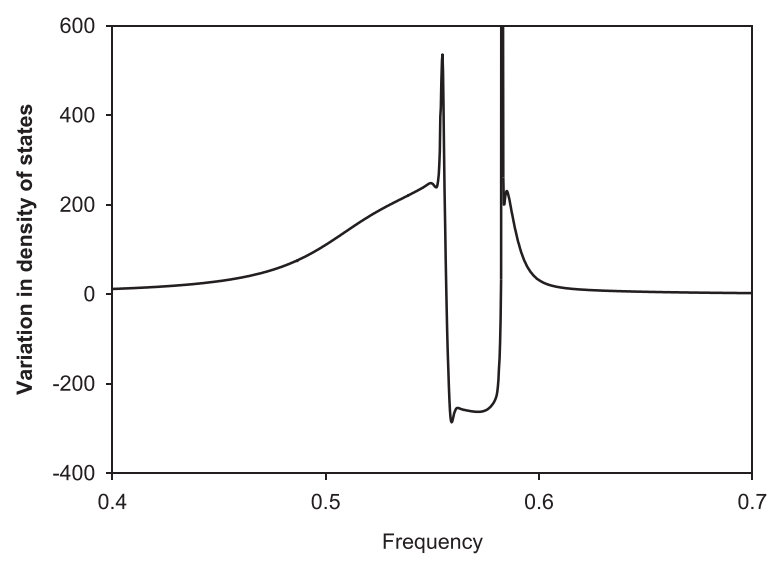

FIG. 4. (a) Variation in density of states, $\Delta n(\omega)$, of the system composed of two parallel chain-like arrangements of adsorbed diatomic molecules containing each 30 molecules, i.e., $N_{c}=60$. The frequency range, $\omega \in[0.4,0.7]$ corresponds to the first resonance in Fig. 2. The resonance frequency of one diatomic molecule is 0.5570 and corresponds to the first sharp peak in the figure.

same frequency. It is the substrate terms in the Green's function matrix [Eq. (15)] contributing through the coupling operator to form the diffusion matrix that lead to this dispersive behavior. The non-separability of the elastic modes of an adsorbed chain is only relative to the elastic states of a configuration composed of well-separated individual adsorbed diatomic molecules. As we noted several times, the dynamical equations (Hamiltonian) of the adsorbed chain system are linear and modes can be expressible as plane waves. The state of the adsorbed loop system is therefore separable as a tensor product of plane wave states. It is known that given a multipartite physical system, whether quantum or classical, the way to subdivide it into subsystems is not unique. ${ }^{11}$ The "gap" is robust as it also appears for configurations derived from the parallel chains but altered such as the configurations with kinks (b), (c), (d), and (e).

The system (a) constitutes our reference system. The phase $\eta(\omega)$ of each configuration (a) through (e) is calculated and corrected for $2 \pi$ discontinuities arising from the numerical calculation. We plot the four differences in corrected phases: $\eta^{(b)}-\eta^{(a)}, \eta^{(c)}-\eta^{(a)}, \eta^{(d)}-\eta^{(a)}$, and $\eta^{(e)}-\eta^{(a)}$ for one, two, three, and four kinks, respectively, in Fig. 5(a). We note that the phase difference decreases monotonically as a function of the number of kinks for frequencies below resonance. A wide range of frequencies below resonance satisfy the rule of phase addition upon successive addition of kinks: $r_{1}=\frac{\eta^{(c)}-\eta^{(a)}}{\eta^{(b)}-\eta^{(a)}} \sim 2, r_{2}=\frac{\eta^{(d)}-\eta^{(a)}}{\eta^{(b)}-\eta^{(a)}} \sim 3$, and $r_{3}=\frac{\eta^{(e)}-\eta^{(a)}}{\eta^{(b)}-\eta^{(a)}} \sim 4$. The first ratio illustrates the phase change upon creating two kinks relative to the phase change upon creation of one kink. The second and third ratios refer to the phase change that occurs when three (four) kinks are created relative to one kink creation. We have first chosen a particular frequency of 0.5305 , for which additive properties of the phase are satisfied. In this case: $\theta_{1}=\eta^{(b)}-\eta^{(a)} \cong-0.2$. In Fig. 5(b), we have plotted the four calculated phase differences in phase as functions of the number of kinks. The calculated points fall on the line with equation $\theta_{n_{k}}=\Delta \theta n_{k}=-0.2 n_{k}$, where $n_{k}$ is the number of kinks. Our choice of frequency was also motivated by the fact that the phase for one kink is the rational

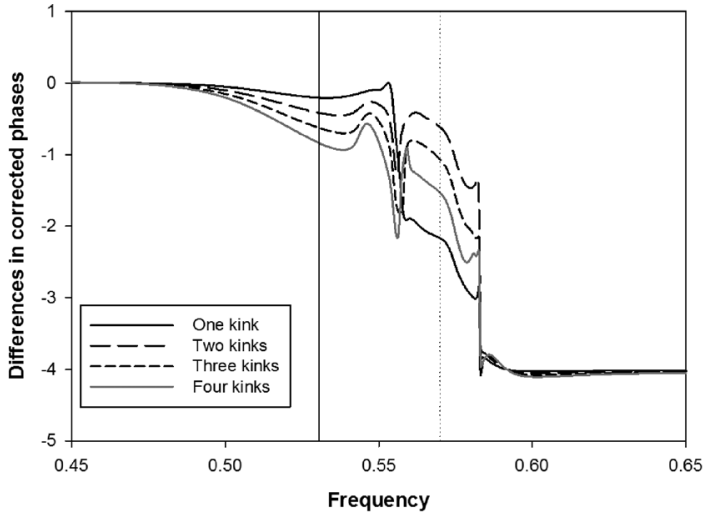

(a)

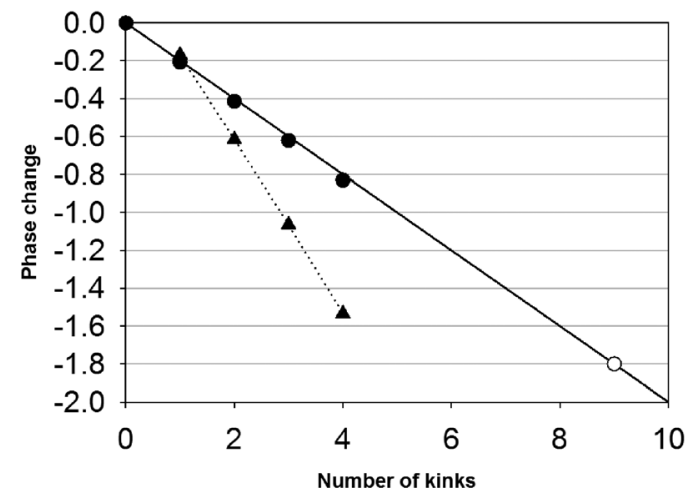

(b)

FIG. 5. (a) Differences in phase $\eta(\omega)$ corrected for $2 \pi$ jumps, relative to the parallel chain system for one, two, three, and four kinks created along two parallel chains of 30 diatomic molecules each. The vertical solid and dotted lines show the frequencies 0.5305 and 0.57. (b) Phase difference from (a) (closed circles) at the frequency 0.5305 and (closed triangles) at the frequency 0.57 as a function of the number of kinks. The thin solid line is a linear fit to the closed circles. The dotted line is a fit to the closed triangles. The open circle marks the location of a chain along which one would create 9 kinks. All the phases are in units of $\pi$. The data point corresponding to one kink at the frequency 0.57 was corrected by a phase shift of 2 to align it with the other points.

quantity $\theta_{1}=\frac{2}{n}$, where $n=10$. Because of computational limitations, we have not been able to consider longer chains and larger numbers of kinks than 4 , however, if one could continue the linear trend of the phase, the addition of kinks would lead to phase differences that sample the unit circle in 10 locations as shown in Fig. 6.

We note that $\theta_{9}=+0.2 \bmod (2)$. The creation of nine kinks is therefore a configuration equivalent of a single kink but with a phase change of the same magnitude but opposite sign.

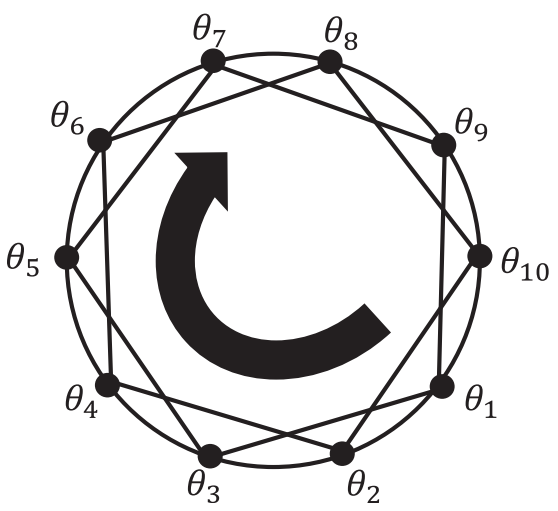

FIG. 6. Schematic illustration of the evolution of the phase difference $\theta_{n_{k}}$ upon creation of $n_{k}$ kinks. 
The elastic wave function of two configurations related by the creation of one kink can be expressed as

$$
u_{n_{k+1}}=e^{i \Delta \theta} u_{n_{k}} .
$$

Note that in Eq. (17), we have assumed that the elastic waves have been normalized to their real amplitude.

The phase difference as a function of number of kink for a frequency of 0.57 is plotted in Fig. 5(b) as triangles. It is interesting to note that the triangular points fall along a straight line which does not intersect the origin. The phase difference follows the equation, $\theta_{n_{k}}=\Delta \theta n_{k}+\delta=-0.4464 n_{b}+0.2769$. The constant phase 0.2769 is not important as it can be lumped as a constant term into the elastic wave functions in Eq. (17).

In Fig. 7, we consider two other frequencies but now slightly above and below the first resonant frequency 0.5570 of the diatomic molecule, namely 0.5577 and 0.5568 . In Fig. 7(a), we show the differences in phases for one to four kinks in a narrow region of frequency centered on the resonant frequency of the diatomic molecule. At 0.5577 , the calculated phase differences for $n_{k}=1$ and 4 have been corrected by $\pm 2(2 \pi)$ to form a continuous function. These corrected points are fitted well by a quadratic equation: $\theta_{n_{k}}=-1.423$ $\times 10^{-1} n_{k}^{2}-3.323 \times 10^{-1} n_{k}+8.105 x \times 10^{-1}$. In contrast to the case of the frequency 0.5305 , this relation is non-linear. One may still write

$$
u_{n_{k+1}}=e^{i \Delta \theta\left(n_{k}\right)} u_{n_{k}}
$$

with a phase shift dependent on the number of kinks, $\Delta \theta\left(n_{k}\right)$.

At the frequency of 0.5568 , below resonance, the behavior of the phase difference is more complex. The calculated phase differences can be corrected by $\pm 2(2 \pi)$ without consequence. The corrected phase differences do not form a continuous function. The data points for $n_{k}=3$ and 4 are $\pi$ shifted above such a continuous line. This additional phase difference $\pi$ is not associated with a transmission phase slip of $\pi$ [as seen in Fig. 2(b)] as the calculated phases in Fig. 7(a) are continuous. This additional $\pi$ shift is a characteristic of the creation of additional kinks beyond 2 kinks and the formation of a finite periodic array of kinks leading to crossings of the phase plots in Fig. $7(\mathrm{a})$. Again, the evolution of the elastic wave function as a function of the number of kinks can be represented by Eq. (18).

We could represent mathematically the relationships given by Eq. (18) in the form of the product between the vector of the elastic waves for different numbers of kinks, $\Psi$ and a unitary matrix, $U$

$$
\begin{aligned}
\Psi & =\left(., u_{i}, u_{i+1}, u_{i+2}, .\right) \\
& =\left(\begin{array}{ccccc}
\cdot & . & . & . & . \\
\cdot & e^{\mathrm{i} \Delta \theta_{i-1}} & 0 & 0 & . \\
\cdot & 0 & e^{\mathrm{i} \Delta \theta_{i}} & 0 & . \\
\cdot & 0 & 0 & e^{\mathrm{i} \Delta \theta_{i+1}} & . \\
\cdot & . & . & . & .
\end{array}\right)\left(\begin{array}{c}
\cdot \\
u_{i-1} \\
u_{i} \\
u_{i+1} \\
\cdot
\end{array}\right)=U \Psi .
\end{aligned}
$$

It is worth noting that at the frequencies 0.5305 and 0.57 , where the accumulation of phase upon creation of additional kinks is a constant (linear dependency of the phase difference on the number of kinks), the diagonal terms in Eq. (19) are all identical. Equation (19) can then be simplified in the form

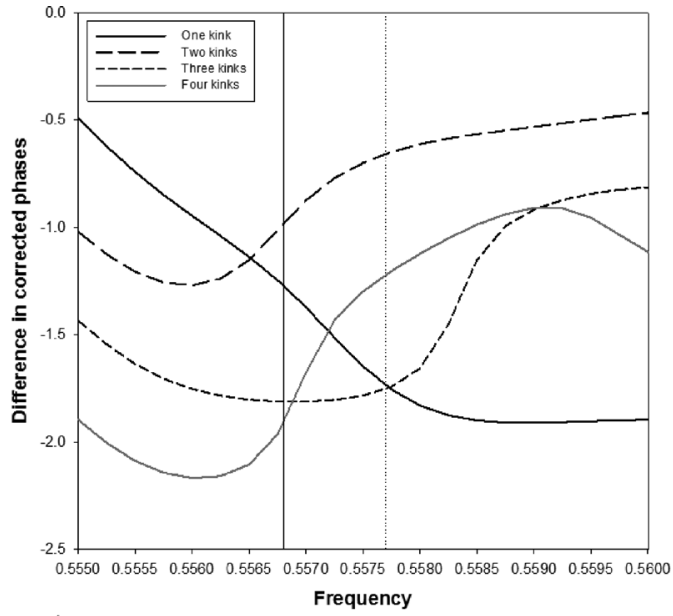

(a)

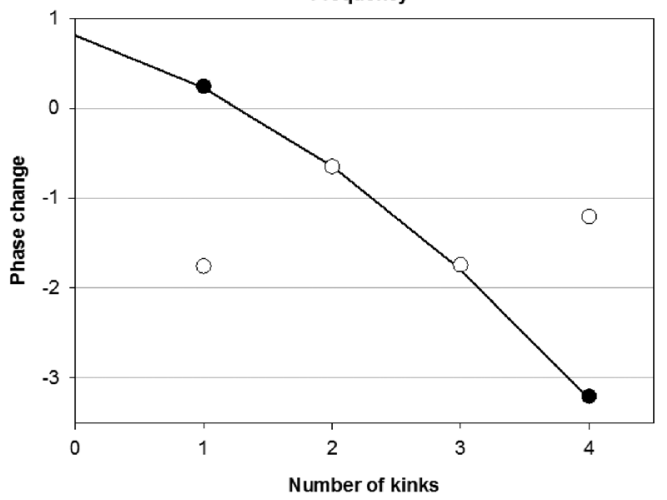

(b)

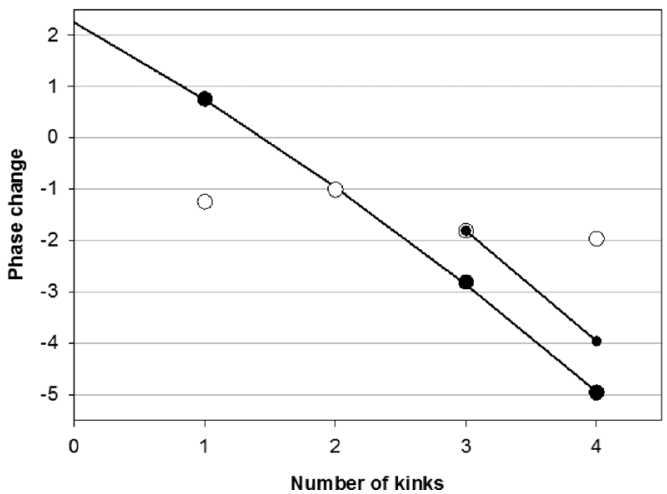

(c)

FIG. 7. (a) Same as Fig. 5(a) but magnified in a narrow frequency range around the diatomic molecule resonant frequency, 0.5570. The black and gray solid vertical lines mark the frequencies 0.5568 and 0.5577 , respectively. (b) Phase difference from (a) (open circles) at the frequency 0.5577 as a function of the number of kinks. The closed circles are corrections by $\pm 2(2 \pi)$ of the phase differences. The thin solid line is a linear fit to the corrected data. (c) Phase difference from (a) (open circles) at the frequency 0.5568 as a function of the number of kinks. The large closed circles are corrections by $\pm 2(2 \pi)$ and $-1(\pi)$ of the phase differences. The thin solid line extending over the interval $[0,4]$ is a linear fit to the corrected data. The small closed circles have been corrected only by $\pm 2(2 \pi)$.

$$
\begin{aligned}
\Psi & =\left(., u_{i}, u_{i+1}, u_{i+2}, .\right) \\
& =\left(\begin{array}{ccccc}
\cdot & \cdot & \cdot & \cdot & . \\
\cdot & 1 & 0 & 0 & . \\
\cdot & 0 & 1 & 0 & . \\
\cdot & 0 & 0 & 1 & . \\
. & . & . & . & .
\end{array}\right) e^{i \Delta \theta}\left(\begin{array}{c}
\cdot \\
u_{i-1} \\
u_{i} \\
u_{i+1} \\
.
\end{array}\right)=I e^{i \Delta \theta} \Psi .
\end{aligned}
$$




\section{Near resonance the phase difference depends on the order of creation of kinks}

To analyze the behavior of the phase in the vicinity of resonance further, we consider three configurations illustrated in Fig. 8. The first configuration includes two parallel chains composed of 13 molecules, that is $12 a$ long. We have chosen an odd number of diatomic molecules to be able to create the configuration of Fig. 8(b) by forming two kinks that are asymmetrical with respect to the marked red line. This enables us to define two types of kinks, the kink to the left of the red line created by an operator $\sigma_{1}$ and the kink to the right of the red line resulting from the operator, $\sigma_{2}$. The configuration in Fig. 8(b) can be visualized as resulting from the application of the sequence $\sigma_{1} \sigma_{2}$ of kink creation operators. The configuration in Fig. 8(c) can be visualized as resulting from the reverse sequence, $\sigma_{2} \sigma_{1}$.

In Fig. 9, we report the calculated phases for these three configurations corrected for numerical jumps of $2 \pi$. However, one can note that the phase near resonance in the case of configuration (c) differs significantly from that of configuration (b).

The phase difference between the kinked configurations and the parallel chains illustrates even more clearly the fact

(a)

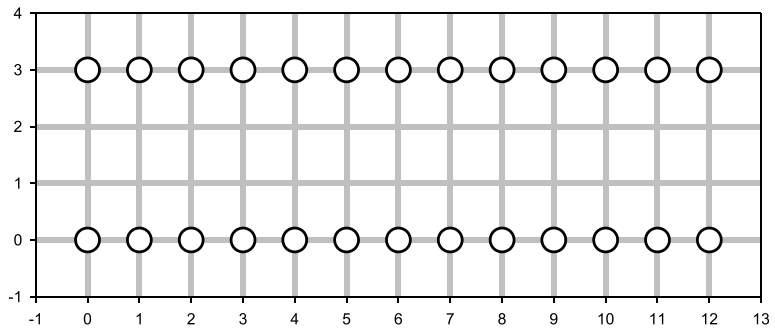

(b)

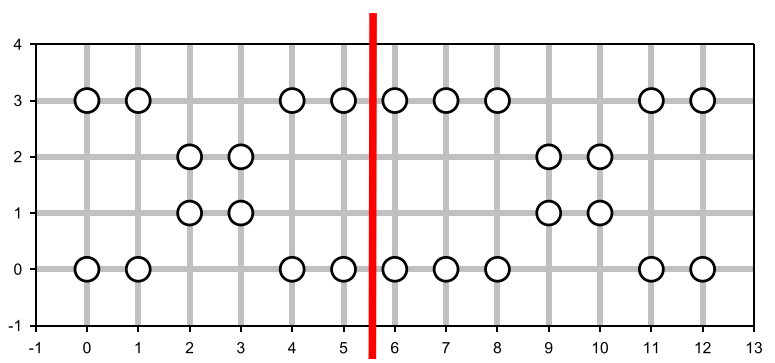

(c)

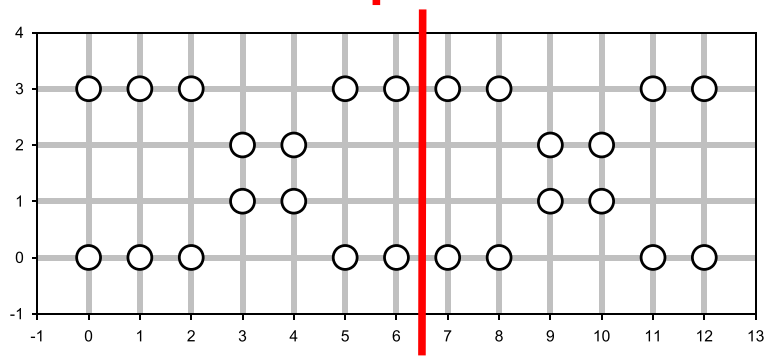

FIG. 8. Top view of three configurations of $N_{c}=26$ diatomic molecules adsorbed on the (001) surface of the simple cubic crystal. The surface is illustrated as a square lattice. (a) Two linear parallel arrays of 13 diatomic molecules. (b) The two linear chains are subjected to the creation of two kinks. The creation of the two kinks is done in sequence from the left to the right. The creation of the first kink to the left of the red line is characterized by an operator, $\sigma_{1}$. The second kink to the right of the red line is created by an operator, $\sigma_{2}$. (c) The two linear chains are subjected to the creation of two kinks by application of the operator, $\sigma_{2}$ first, followed by the creation of the second kink by applying $\sigma_{1}$. In (b), the order of creation from left to right is $\left(\sigma_{1} \sigma_{2}\right)$ and $\left(\sigma_{2} \sigma_{1}\right)$ in $(\mathrm{c})$.

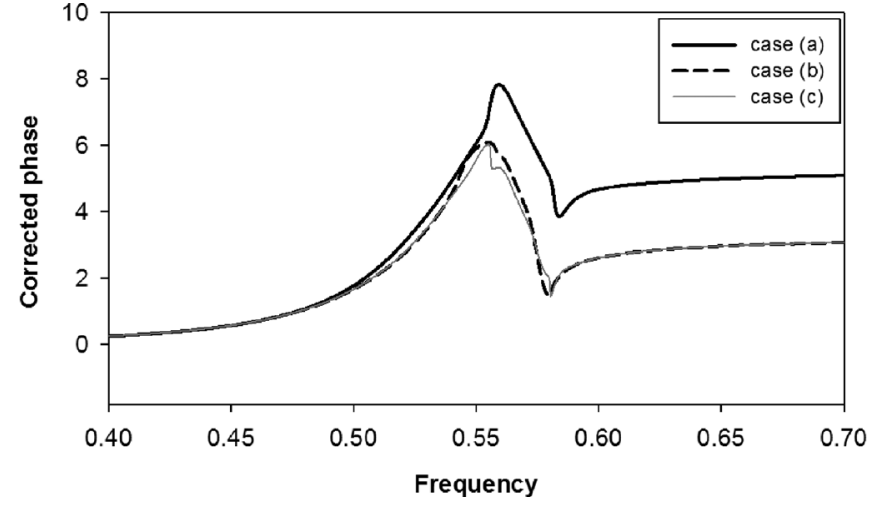

FIG. 9. Phase $\eta(\omega)$ corrected for $2 \pi$ jumps for the three configurations (a), (b), and (c), illustrated in Fig. 8.

that the two configurations (b) and (c) do not exhibit the same phase around the resonance (see Fig. 10). The phase differences do show the same phase behavior at frequencies above and below resonance frequency. This clearly shows that if we consider different kink creation operators, $\sigma_{1}$ and $\sigma_{2}$, near resonance, the order application of these operations matters. We may therefore write that $\sigma_{1} \sigma_{2} \neq \sigma_{2} \sigma_{1}$. Near resonance, the order of creation of different kinks matters, the kink creation operators do not generally commute.

\section{Chains of diatomic molecules adsorbed on the surface of a cubic crystal along the (110) direction}

In Subsection III C, we considered chains aligned in the (100) direction in the surface square lattice. However, there are other ways of construction chains on that lattice. One of these directions is the (110) direction. In Fig. 11, we consider four new configurations of adsorbed diatomic molecules on the crystal surface. The first configuration consists of two parallel arrays of molecules arranged along the diagonal of the unit cell of the two-dimensional lattice of the surface. Each array contains 20 molecules. We consider other configurations by applying a series of kink creation operations. For instance, one creates one, two, and three kinks in configurations (b), (c), and (d).

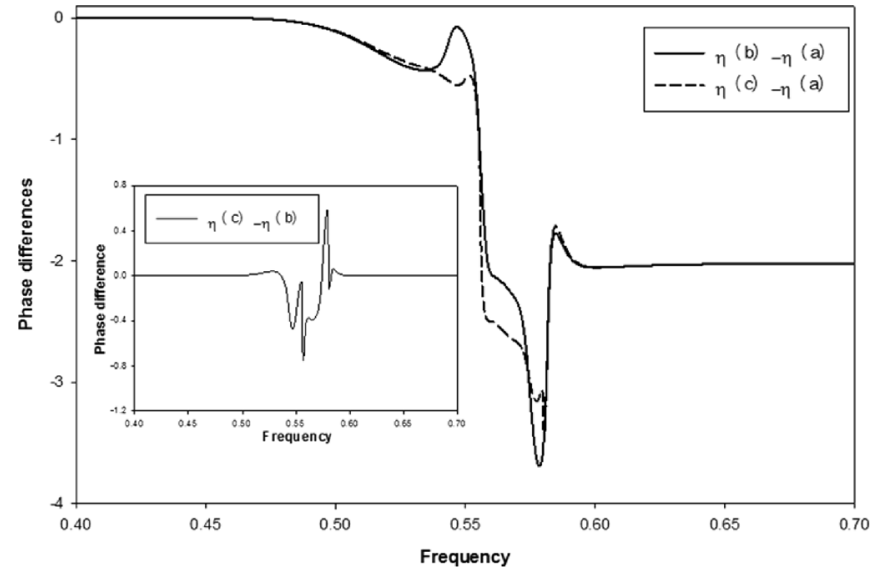

FIG. 10. Differences between the corrected phase of configurations (b) and (c) and the reference system [configuration (a)] as functions of frequency. The inset illustrates the difference in phase between the two kinked configurations (b) and (c). 


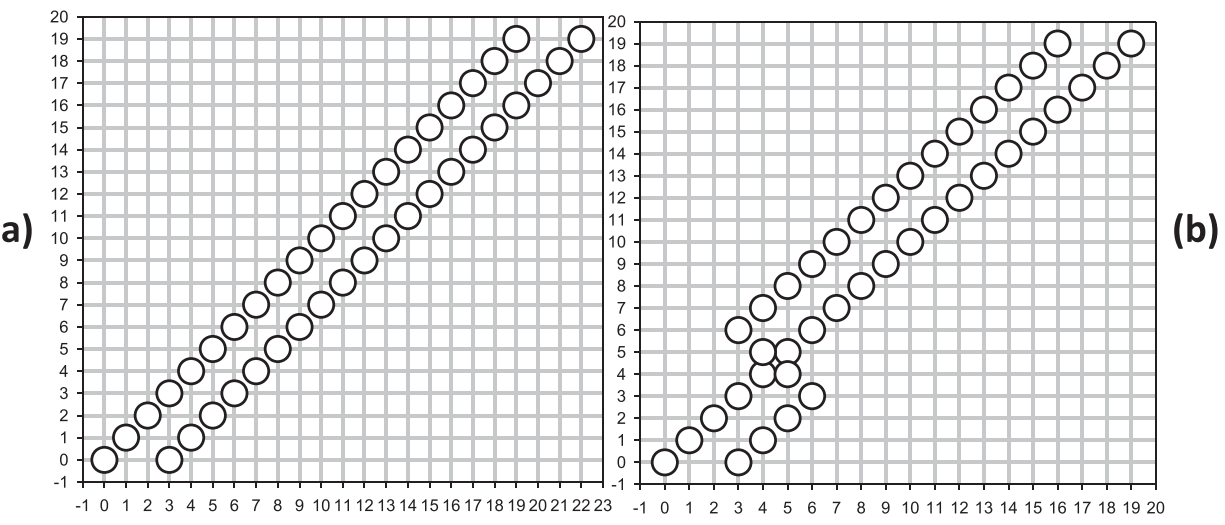

(b)
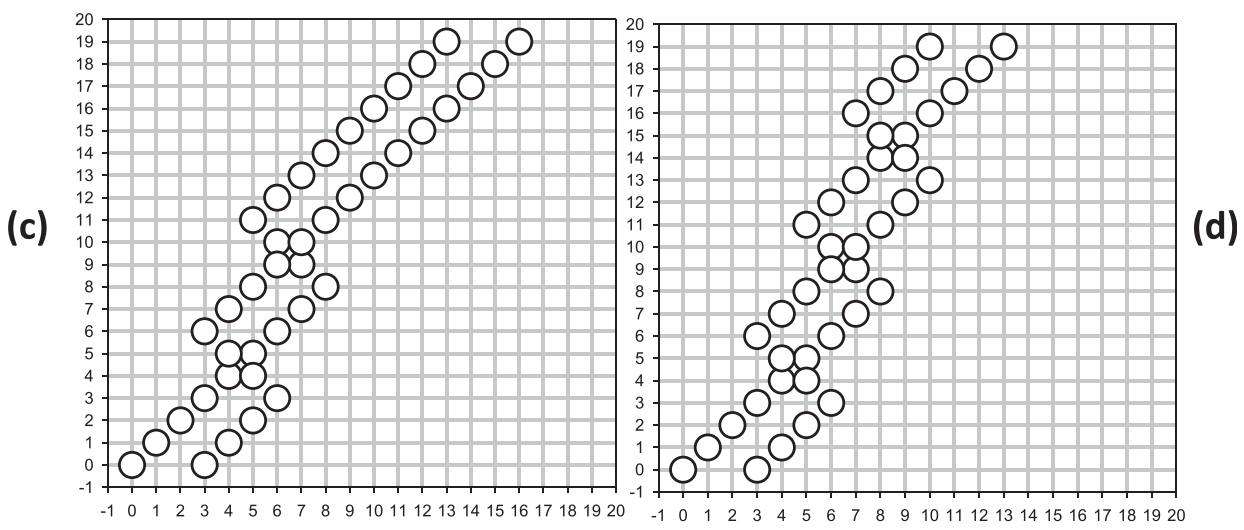

FIG. 11. Top view of the four configurations of $N_{c}=40$ diatomic molecules adsorbed on the (001) surface of the simple cubic crystal. (a) Two linear parallel arrays of diatomic molecules arranged along the (110) direction. The chains are separated by a distance exceeding the Green's function cut off distance $\sqrt{5} a$. (b) The two linear chains are subjected to one kink creation operation. Note that in the process of creating a kink, the overall location of the ends is translated but their relative position is not. (c) The two linear chains are subjected to two successive kink creation operations. (d) The two linear arrays support three kinks.

In Fig. 12, we report the phase for the configurations (a), (b), (c), and (d) as functions of frequency. These phases have been corrected for numerically induced $2 \pi$ discontinuous jumps.

The corrected phases differ most significantly in the near vicinity of the resonance. Above resonance, the phase of configurations (c) and (d) are $2 \pi$ shifted relative to that of the configurations (a) and (b). After correcting for this shift, we report in Figs. 13(a) and 13(b), the ratios: $r_{1}=\frac{\eta^{(c)}-\eta^{(a)}}{\eta^{(b)}-\eta^{(a)}}$ and $r_{2}=\frac{\eta^{(d)}-\eta^{(a)}}{\eta^{(b)}-\eta^{(a)}}$ as functions of frequency.

Here, as was the case for chains along the (100) direction, the phase of parallel chains along the (110) direction increases by a multiple for every kink created. We note that

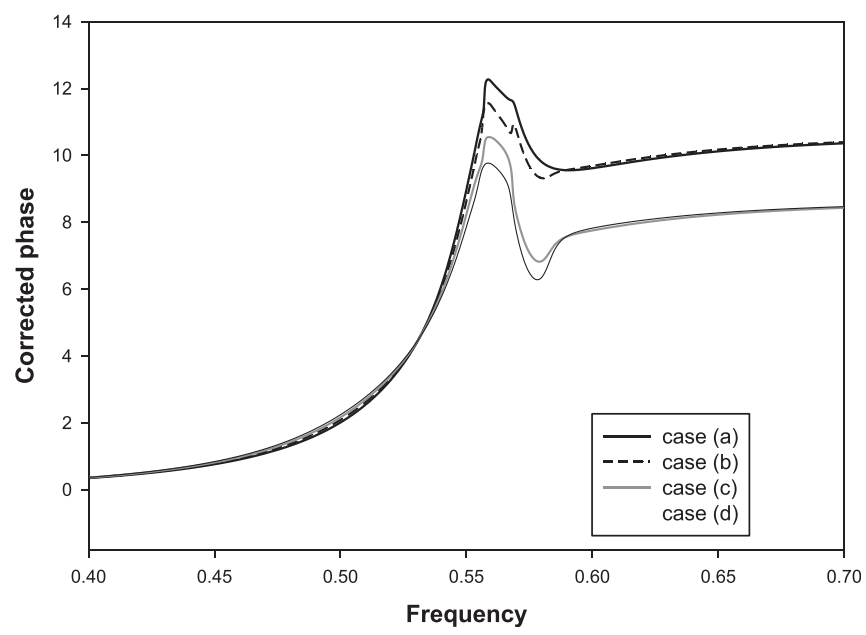

FIG. 12. Phase $\eta(\omega)$ corrected for $2 \pi$ jumps for the four configurations (a), (b), (c), and (d), illustrated in Fig. 11. well below resonance and well above resonance, the ratios $r_{1}$ and $r_{2}$ take on the values 2 and 3 , that is, the phase of the system with two kinks is twice that of the system with one kink and the phase with three kinks is three times that of one kink. This behavior leads to linear variation of the phase differences with respect to the number of kinks. The phase difference behaves according to Eq. (20). We note that in Fig. 13, there are several frequencies for which peculiar behavior is observed. There exist a number of frequencies outside resonance where $\eta^{(b)}-\eta^{(a)} \cong 0$, thus leading also to diverging ratios, ratios $r_{1}$ and $r_{2}$. Two examples are located at frequencies 0.531 and 0.589 , where from Fig. 12 , one clearly sees crossing of the phase of the configurations (a) and (b).

In Fig. 14, we plot the difference in phases of configurations with one, two, and three kinks with respect to the parallel configuration at the three frequencies $0.5052,0.5599$, and 0.5700 . Figure 14 also includes linear fits to the numerical data points. The fits are $\theta_{n_{k}}(0.5052)=7.865 \times 10^{-2} n_{k}+1$ $\times 10^{-4}, \theta_{n_{k}}(0.5599)=-8.736 \times 10^{-1} n_{k}+1.313 \times 10^{-1}$, and $\theta_{n_{b}}(0.5700)=-5.231 \times 10^{-1} n_{k}-5.97 \times 10^{-2}$. The intercept for the lowest frequency is within the error of our calculations and the phase difference intersects the vertical axis at the origin. The other two cases have finite intercepts but are still showing nearly linear behavior compatible with Eq. (20).

At the resonant frequency, 0.5570 , the phase difference as a function of the number of kinks shows strong non-linear dependency (Fig. 15) which indicates that the elastic wave function follow Eq. (19). We constructed the concave plot in Fig. 15 by applying a $-2 \pi$ correction to the last data point (three kinks). Even in the absence of this correction, the plot would be convex and non-linear. 


\section{ANALOGY WITH QUANTUM GATES}

Here, we consider, analogies between the phase-based operations that could be accomplished with unitary operations resulting from the creation of kinks in parallel chains of diatomic molecules adsorbed on a simple cubic crystal surface. Quantum gates (or quantum logic gates) are the elementary building blocks of quantum circuits. Quantum gates are represented by unitary matrices which act on the phase of quantum states. ${ }^{12}$ The fact that one can transform the phase of elastic waves by modifying the configuration of adsorbed diatomic molecules, and that these transformations can be formulated as unitary matrices acting on the space of the scattered waves, enables us to draw analogies between elastic phase-based unitary transformations and quantum gates.

Let us consider the configurations introduced in Sec. III B. At the frequency 0.5577 , the phase accumulation as a function of the number of kinks can be reformulated according to Eq. (19)

$$
\begin{aligned}
\left(u_{1}, u_{2}, u_{3}, u_{4}, .\right) & =\left(\begin{array}{cccccc}
e^{\mathrm{i} \Delta \theta_{0}} & 0 & 0 & 0 & \cdot \\
0 & e^{\mathrm{i} \Delta \theta_{1}} & 0 & 0 & \cdot \\
0 & 0 & e^{\mathrm{i} \Delta \theta_{2}} & 0 & \cdot \\
0 & 0 & 0 & e^{\mathrm{i} \Delta \theta_{3}} & \cdot \\
\cdot & \cdot & \cdot & . & \cdot
\end{array}\right)\left(\begin{array}{c}
u_{0} \\
u_{1} \\
u_{2} \\
u_{3} \\
\cdot
\end{array}\right) \\
& =\left(\begin{array}{ccccc}
1 & 0 & 0 & 0 & . \\
0 & e^{\mathrm{i} \varphi_{1}} & 0 & 0 & \cdot \\
0 & 0 & e^{\mathrm{i} \phi_{2}} & 0 & \cdot \\
0 & 0 & 0 & e^{\mathrm{i} \phi_{3}} & \cdot \\
. & . & . & . & .
\end{array}\right) e^{\mathrm{i} \Delta \theta_{0}}\left(\begin{array}{c}
u_{0} \\
u_{1} \\
u_{2} \\
u_{3} \\
\cdot
\end{array}\right)
\end{aligned}
$$

with $\varphi_{i}=\Delta \theta_{i}-\Delta \theta_{0}$.

At the frequency of 0.5568 , we have

$$
\begin{aligned}
& \left(u_{1}, u_{2}, u_{3}, u_{4}, .\right)=\left(\begin{array}{ccccc}
e^{\mathrm{i} \Delta \theta_{0}} & 0 & 0 & 0 & \cdot \\
0 & e^{\mathrm{i} \Delta \theta_{1}} & 0 & 0 & \cdot \\
0 & 0 & e^{\mathrm{i}\left(\Delta \theta_{2}+\pi\right)} & 0 & . \\
0 & 0 & 0 & e^{\mathrm{i}\left(\Delta \theta_{3}+\pi\right)} & . \\
. & . & . & . & .
\end{array}\right)\left(\begin{array}{c}
u_{0} \\
u_{1} \\
u_{2} \\
u_{3} \\
\cdot
\end{array}\right) \\
& =\left(\begin{array}{ccccc}
e^{\mathrm{i} \Delta \theta_{0}} & 0 & 0 & 0 & \cdot \\
0 & e^{\mathrm{i} \Delta \theta_{1}} & 0 & 0 & \cdot \\
0 & 0 & e^{\mathrm{i} \Delta \theta_{2}} & 0 & . \\
0 & 0 & 0 & e^{\mathrm{i} \Delta \theta_{3}} & \cdot \\
. & . & . & \cdot & .
\end{array}\right)\left(\begin{array}{ccccc}
1 & 0 & 0 & 0 & \cdot \\
0 & 1 & 0 & 0 & \cdot \\
0 & 0 & -1 & 0 & \cdot \\
0 & 0 & 0 & -1 & \cdot \\
\cdot & \cdot & . & . & .
\end{array}\right)\left(\begin{array}{c}
u_{0} \\
u_{1} \\
u_{2} \\
u_{3} \\
\cdot
\end{array}\right) \\
& =\left(\begin{array}{ccccc}
1 & 0 & 0 & 0 & . \\
0 & e^{\mathrm{i} \varphi_{1}} & 0 & 0 & . \\
0 & 0 & e^{\mathrm{i} \phi_{2}} & 0 & . \\
0 & 0 & 0 & e^{\mathrm{i} \phi_{3}} & . \\
. & . & . & . & .
\end{array}\right) e^{\mathrm{i} \Delta \theta_{0}}\left(\begin{array}{ccccc}
1 & 0 & 0 & 0 & . \\
0 & 1 & 0 & 0 & . \\
0 & 0 & -1 & 0 & . \\
0 & 0 & 0 & -1 & . \\
. & . & . & . & .
\end{array}\right)\left(\begin{array}{c}
u_{0} \\
u_{1} \\
u_{2} \\
u_{3} \\
\cdot
\end{array}\right) .
\end{aligned}
$$

The unitary transformation matrices are therefore rewritable in the form of products of quantum gates.

For instance, focusing on only two kink creation operations, Eq. (21) may be reduced to

$$
\left(u_{1}, u_{2}, .\right)=\left(\begin{array}{ccccc}
1 & 0 & . & . & . \\
0 & e^{\mathrm{i} \varphi_{1}} & . & . & . \\
. & . & . & . & . \\
\cdot & . & . & . & . \\
. & . & . & . & .
\end{array}\right) e^{\mathrm{i} \Delta \theta_{0}}\left(\begin{array}{c}
u_{0} \\
u_{1} \\
\cdot \\
\cdot \\
.
\end{array}\right) .
$$

This unitary matrix of Eq. (23) can act as a phase shift gate: $R_{\varphi}=\left(\begin{array}{cc}1 & 0 \\ 0 & e^{i \varphi}\end{array}\right)$. The unitary matrix of Eq. (22) may be rewritten as

$$
\begin{aligned}
\left(u_{1}, u_{2}, u_{3}, u_{4}, .\right)= & \left(\begin{array}{ccccc}
e^{\mathrm{i} \varphi_{0}^{\prime}} & 0 & 0 & 0 & . \\
0 & 1 & 0 & 0 & . \\
0 & 0 & e^{\mathrm{i} \varphi_{2}^{\prime}} & 0 & . \\
0 & 0 & 0 & e^{\mathrm{i} \varphi_{3}^{\prime}} & . \\
\cdot & \cdot & . & . & .
\end{array}\right) \\
& \times e^{\mathrm{i} \Delta \theta_{1}}\left(\begin{array}{ccccc}
1 & 0 & 0 & 0 & . \\
0 & 1 & 0 & 0 & . \\
0 & 0 & -1 & 0 & . \\
0 & 0 & 0 & -1 & . \\
. & \cdot & . & . & .
\end{array}\right)\left(\begin{array}{c}
u_{0} \\
u_{1} \\
u_{2} \\
u_{3} \\
\cdot
\end{array}\right)
\end{aligned}
$$

with $\varphi_{i}^{\prime}=\Delta \theta_{i}-\Delta \theta_{1}$ or 
(a)

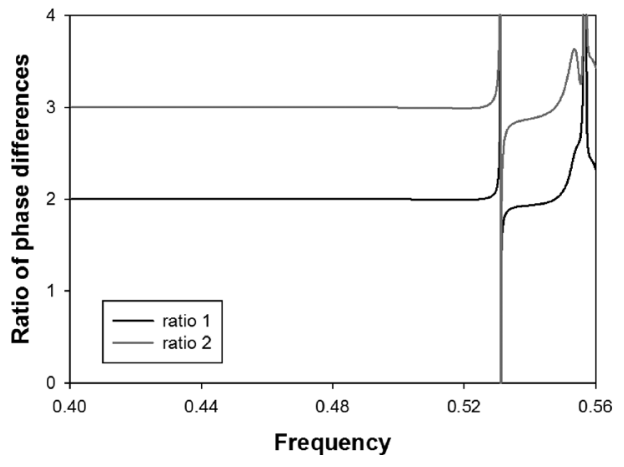

$$
\begin{aligned}
& \left(., u_{2}, u_{3}, .\right)=\left(\begin{array}{ccccc}
\cdot & . & . & . & . \\
\cdot & 1 & 0 & 0 . & . \\
\cdot & 0 & e^{\mathrm{i} \varphi_{2}^{\prime}} & . & . \\
\cdot & \cdot & . & . & . \\
\cdot & . & . & . & .
\end{array}\right) \\
& \times e^{\mathrm{i} \Delta \theta_{1}}\left(\begin{array}{ccccc}
\cdot & \cdot & . & . & . \\
\cdot & 1 & 0 & . & . \\
\cdot & 0 & -1 & . & . \\
\cdot & \cdot & . & . & \cdot \\
\cdot & . & . & . & .
\end{array}\right)\left(\begin{array}{c}
\cdot \\
u_{1} \\
u_{2} \\
\cdot \\
\cdot
\end{array}\right)
\end{aligned}
$$

Equation (25) involves the product of a phase shift gate and Pauli $Z$ gate: $Z=\left(\begin{array}{cc}1 & 0 \\ 0 & -1\end{array}\right)$.

While realizing the $R_{\varphi}$ and $\mathrm{Z}$ gates is useful, the possibility of realizing general unitary operations given by a matrix $U=\left(\begin{array}{ccccc}e^{\mathrm{i} \Delta \theta_{0}} & 0 & 0 & 0 & . \\ 0 & e^{\mathrm{i} \Delta \theta_{1}} & 0 & 0 & . \\ 0 & 0 & e^{\mathrm{i} \Delta \theta_{2}} & 0 & . \\ 0 & 0 & 0 & e^{\mathrm{i} \Delta \theta_{3}} & . \\ . & . & . & . & .\end{array}\right)$ creates novel opportunities for using classical elastic waves for phasebased information processing.

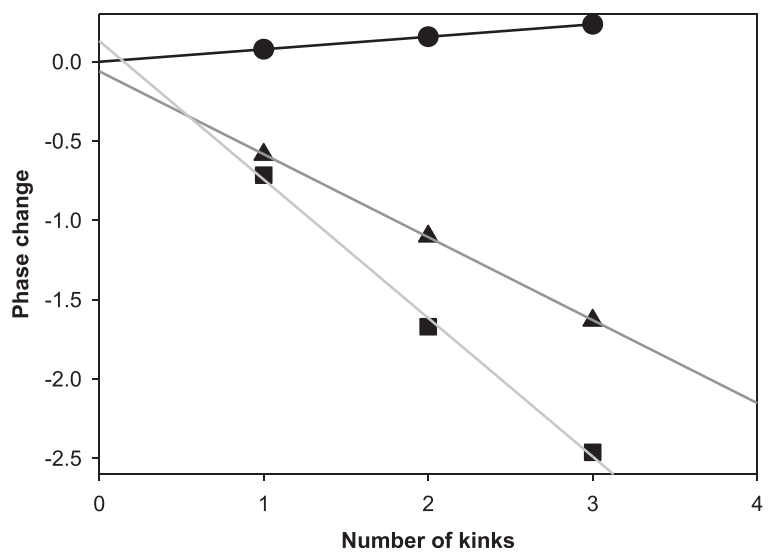

FIG. 14. Phase difference as a function of number of kinks for three frequencies: 0.5052 (circles), 0.5599 (squares), and 0.57 (triangles). The black, dark gray, and light gray solid lines are a linear fit to the circles, squares, and triangles. All the phases are in units of $\pi$. (b)

FIG. 13. Ratios $r_{1}$ and $r_{2}$ as functions of frequency at frequencies below resonance (a) and above resonance (b). The phase differences above resonance have been corrected by $2 \pi$ prior to calculating the ratios.

These gates are therefore realizable physically by considering a number of couples of parallel chains of diatomic molecules adsorbed on the surface of the cubic crystal (e.g., parallel to the (100) direction) on which one can operate by moving molecules to form the desired number of kinks.

\section{CONCLUSIONS}

We have developed an elastic model of diatomic molecules adsorbed on the (001) surface of a simple cubic crystal. We demonstrate that the phase of elastic waves scattered by some number of adsorbed diatomic molecules depends on the particular features of the configuration of the molecules. For instance, we consider parallel arrays of chain-like configurations of the molecules. These parallel arrays are further modified by creating kinks along their length. At frequencies deviating from a resonant frequency of the diatomic molecules, the phase of scattered elastic waves scales linearly with the number of kinks created. However, in the near vicinity of a resonant frequency, the phase does not scale linearly anymore but exhibits nonlinear dependency on the number of kinks. We also show that near a resonance frequency, the order of creation of kinks with different symmetry affects the final value of the phase of scattered waves. The effect of the creation of kinks along the parallel chains can be formulated mathematically as unitary matrix transformations acting on vectors in the space of elastic scattered waves. Analogies are drawn between these phase-based

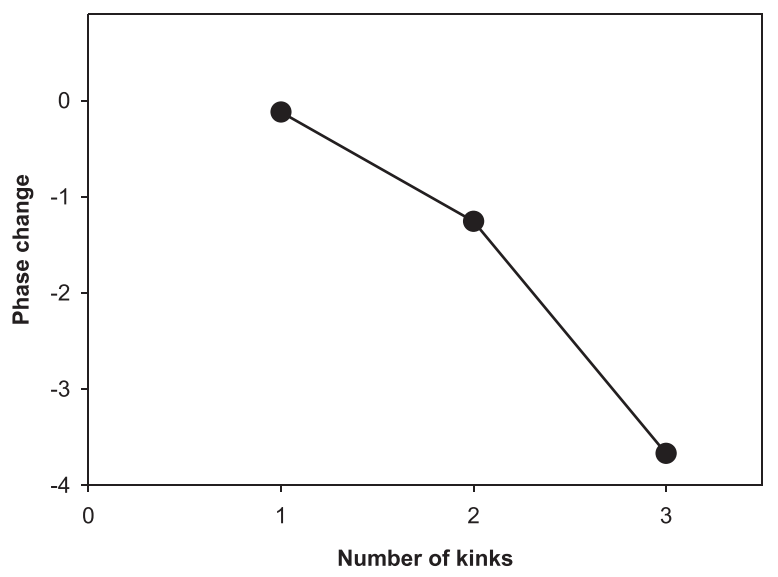

FIG. 15. Phase difference as a function of number of kinks for the resonant frequency. All the phases are in units of $\pi$. The last data point was corrected by an amount of $-2 \pi$. 
elastic unitary matrices and quantum gates. The advantage of elastic based unitary gates is that they are realizable physically by considering a number of couples of parallel chains of diatomic molecules adsorbed on the surface of the cubic crystal (e.g., parallel to the (001) direction) on which one can operate by moving molecules to form the desired number of kinks. Displacing molecules on the surface of a crystal can be achieved using a scanning tunneling microscope. ${ }^{13}$ However, experimental measurement of the phase of the vibrational modes of the adsorbed molecules will remain a challenge. The challenge of working with molecular size objects can be alleviated by realizing that the systems studied here are discrete metaphors of continuous systems. Indeed, one may consider configurations as simple as patterns of stubs attached to the planar surface of a solid substrate. ${ }^{14}$ The stubs play the role of the diatomic molecules as they also possess a number of discrete resonant modes. A number of stubs may be arranged in configurations similar to those investigated here, namely parallel chains of stubs or chains with kinks. The coupling between stubs is now achieved through the continuous substrate. Macroscale systems will enable the characterization of the vibrational modes and their phase using well-established techniques such as laser Doppler vibrometry.
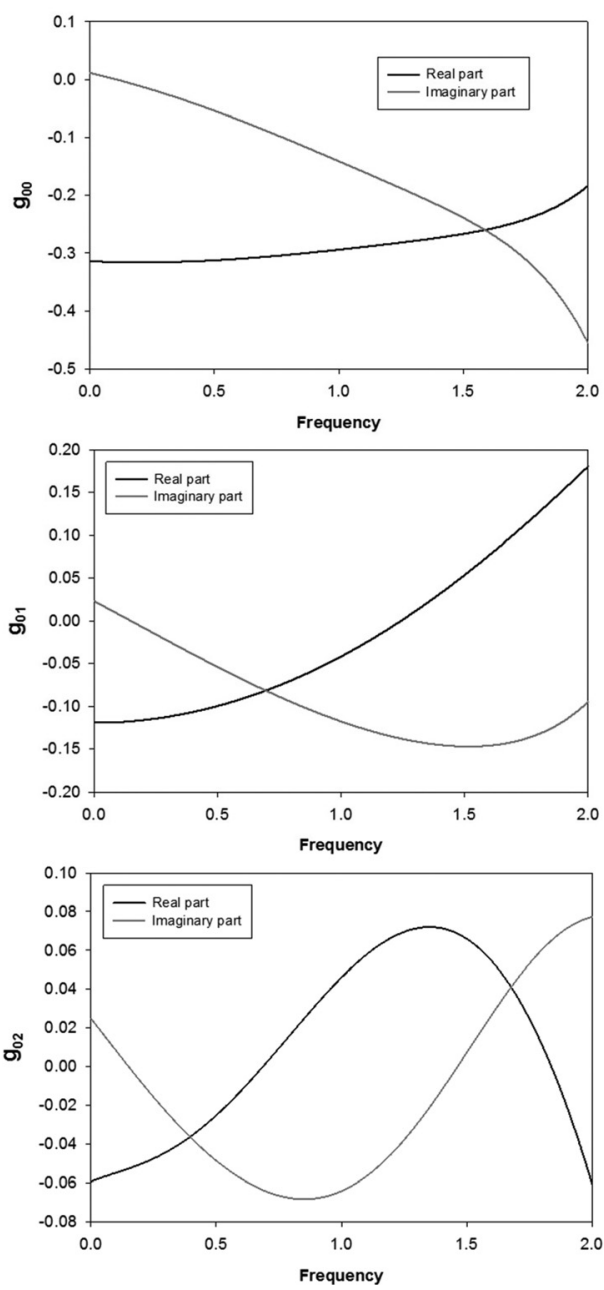

\section{ACKNOWLEDGMENTS}

We acknowledge financial support from the NSF Award No. 1640860 and from the W. M. Keck Foundation.

\section{APPENDIX: GREEN'S FUNCTIONS ON (001) SURFACE OF A CUBIC CRYSTAL}

The fits to the two-dimensional Fourier transform of the Green's functions on the (001) surface of a simple cubic crystal [Eqs. (5) and (6)]: $g_{S 1}\left(\left|p_{i}-p_{j}\right|=0, \omega\right)=g_{00}$, $g_{S 1}\left(\left|p_{i}-p_{j}\right|=1 a, \omega\right)=g_{01}, \quad g_{S 1}\left(\left|p_{i}-p_{j}\right|=2 a, \omega\right)=g_{02}$, $g_{S 1}\left(\left|p_{i}-p_{j}\right|=\sqrt{2} a, \omega\right)=g_{11}, \quad$ and $\quad g_{S 1}\left(\left|p_{i}-p_{j}\right|=\sqrt{5} a, \omega\right)$ $=g_{12}$ and given and plotted below (Fig. 16). The twodimensional Fourier transforms are calculated for 512 values of $k_{1}$ and $k_{2}$.

The real part and imaginary parts of the functions $g_{00}$ and $g_{01}$ are given as $g_{i j}=\left(A_{R, I} \omega^{4}+B_{R, I} \omega^{3}+C_{R, I} \omega^{2}\right.$ $\left.+D_{R, I} \omega+E_{R, I}\right) e^{\left(F_{R, I} \omega+H_{R, I}\right)}$ with the coefficients

$$
\begin{aligned}
& A_{R}^{00}=2.411 \times 10^{-5}, \quad B_{R}^{00}=9.376 \times 10^{-2}, \\
& C_{R}^{00}=-5.348 \times 10^{-1}, \quad D_{R}^{00}=1.184 \times 10^{0}, \\
& E_{R}^{00}=-1.034 \times 10^{0}, \quad F_{R}^{00}=1.202 \times 10^{0}, \quad \text { and } \\
& H_{R}^{00}=-1.191 \times 10^{0},
\end{aligned}
$$
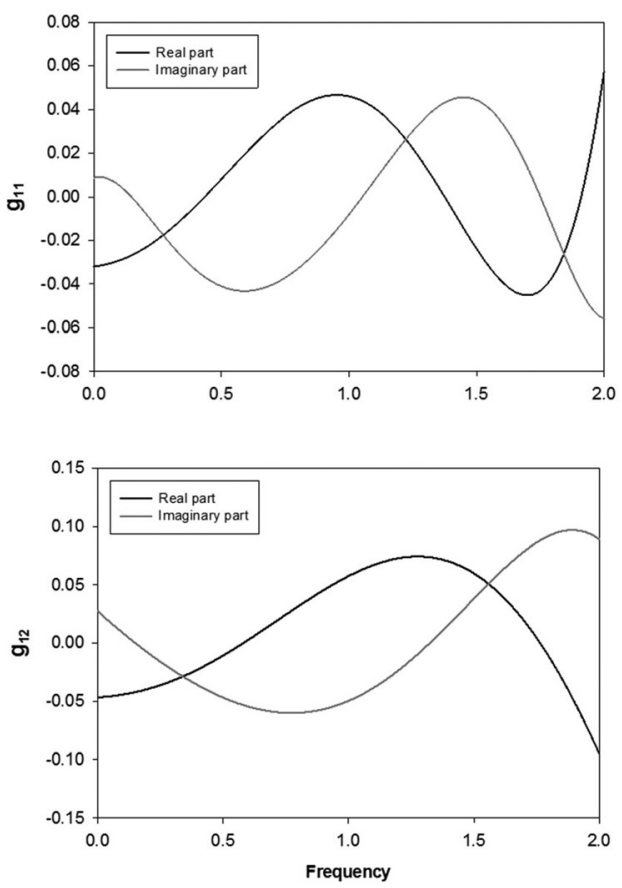

FIG. 16. Fits to the numerically calculated Fourier transforms of the Green's functions $g_{00}, g_{01}, g_{02}, g_{11}$, and $g_{12}$ as functions of frequency. The Green's functions are in units of $\frac{m}{\beta}$. 
and

$$
\begin{aligned}
& A_{I}^{00}=-1.282 \times 10^{-6}, \quad B_{I}^{00}=-6.859 \times 10^{-1}, \\
& C_{I}^{00}=2.854 \times 10^{0}, \quad D_{I}^{00}=-3.528 \times 10^{0}, \\
& E_{I}^{00}=3.402 \times 10^{-1}, \quad F_{I}^{00}=1.429 \times 10^{0}, \quad \text { and } \\
& H_{I}^{00}=-3.407 \times 10^{0},
\end{aligned}
$$

and

$$
\begin{aligned}
& A_{R}^{01}=2.561 \times 10^{-1}, \quad B_{R}^{01}=1.559 \times 10^{-1}, \\
& C_{R}^{01}=5.027 \times 10^{-1}, \quad D_{R}^{01}=-6.240 \times 10^{-1}, \\
& E_{R}^{01}=-9.101 \times 10^{-1}, \quad F_{R}^{01}=-6.644 \times 10^{-1}, \quad \text { and } \\
& H_{R}^{01}=-2.033 \times 10^{0},
\end{aligned}
$$

and

$$
\begin{array}{ll}
A_{I}^{01}=4.647 \times 10^{-2}, & B_{I}^{01}=-3.046 \times 10^{-1}, \\
C_{I}^{01}=7.857 \times 10^{-1}, & D_{I}^{01}=-7.979 \times 10^{-1}, \\
E_{I}^{01}=1.025 \times 10^{-1}, & F_{I}^{01}=1.128 \times 10^{0}, \quad \text { and } \\
H_{I}^{01}=-1.483 \times 10^{0} . &
\end{array}
$$

The real part and imaginary parts of the function $g_{02}$ are given as $\operatorname{Reg}_{02}=\left(A_{R} \omega^{4}+B_{R} \omega^{3}+C_{R} \omega^{2}+D_{R} \omega+E_{R}\right) e^{\left(F_{R} \omega+H_{R}\right)}$ and $\operatorname{Img}_{02}=A_{I} \cos \left(B_{I} \omega+C_{I}\right)+D_{I} \cos \left(E \omega+F_{I}\right)$ with the coefficients

$$
\begin{array}{ll}
A_{R}^{02}=-1.794 \times 10^{0}, & B_{R}^{02}=3.793 \times 10^{0}, \\
C_{R}^{02}=-8.232 \times 10^{-1}, & D_{R}^{02}=1.227 \times 10^{-1}, \\
E_{R}^{02}=-5.249 \times 10^{-1}, & F_{R}^{02}=-6.415 \times 10^{-1}, \quad \text { and } \\
H_{R}^{02}=-2.180 \times 10^{0} &
\end{array}
$$

and

$$
\begin{aligned}
& A_{I}^{02}=5.675 \times 10^{-3}, \quad B_{I}^{02}=4.733 \times 10^{0}, \\
& C_{I}^{02}=-3.170 \times 10^{0}, \quad D_{I}^{02}=7.259 \times 10^{-2}, \\
& E_{I}^{02}=2.488 \times 10^{0}, \quad \text { and } \quad F_{I}^{02}=1.136 \times 10^{0} .
\end{aligned}
$$

The real part and imaginary parts of the functions $g_{11}$ and $g_{12}$ are given as $g_{i j}=A_{R, I} \omega^{5}+B_{R, I} \omega^{4}+C_{R, I} \omega^{3}+D_{R, I} \omega^{2}$ $+E_{R, I} \omega+F_{R, I}$ with the coefficients

$$
\begin{array}{ll}
A_{R}^{11}=-4.480 \times 10^{-2}, & B_{R}^{11}=1.839 \times 10^{-1}, \\
C_{R}^{11}=-2.957 \times 10^{-1}, & D_{R}^{11}=2.983 \times 10^{-1}, \\
E_{R}^{11}=-4.589 \times 10^{-2}, & \text { and } \quad F_{R}^{11}=-7.422 \times 10^{-2},
\end{array}
$$

and

$$
\begin{aligned}
& A_{I}^{11}=9.447 \times 10^{-2}, \quad B_{I}^{11}=-4.220 \times 10^{-1}, \\
& C_{I}^{11}=7.380 \times 10^{-1}, \quad D_{I}^{11}=-5.100 \times 10^{-1}, \\
& E_{I}^{11}=-6.443 \times 10^{-3}, \quad \text { and } \quad F_{I}^{11}=9.249 \times 10^{-3},
\end{aligned}
$$

and

$$
\begin{array}{ll}
A_{R}^{12}=2.938 \times 10^{-2}, & B_{R}^{12}=-1.424 \times 10^{-1}, \\
C_{R}^{12}=1.126 \times 10^{-1}, & D_{R}^{12}=8.992 \times 10^{-2}, \\
E_{R}^{12}=1.476 \times 10^{-2}, & \text { and } \quad F_{R}^{12}=-4.674 \times 10^{-2},
\end{array}
$$

and

$$
\begin{array}{ll}
A_{I}^{12}=-4.263 \times 10^{-2}, & B_{I}^{12}=1.152 \times 10^{-1}, \\
C_{I}^{12}=-5.344 \times 10^{-2}, & D_{I}^{12}=1.013 \times 10^{-1}, \\
E_{I}^{12}=-1.977 \times 10^{-1}, & \text { and } \quad F_{I}^{12}=2.756 \times 10^{-2} .
\end{array}
$$

${ }^{1}$ Acoustic Metamaterials and Phononic Crystals, Springer Series in Solid State Sciences Vol. 173, edited by P. A. Deymier (Springer, Berlin, 2013). ${ }^{2}$ P. A. Deymier and K. Runge, Sound Topology, Duality, Coherence and Wave-Mixing: An Introduction to the Emerging New Science of Sound, Springer Series in Solid State Sciences Vol. 188 (Springer, Berlin, 2017).

${ }^{3}$ M. V. Berry, "Quantal phase factors accompanying adiabatic changes," Proc. R. Soc. A 392, 45 (1984).

${ }^{4} \mathrm{~J}$. Friedel, "The distribution of electrons round impurities in monovalent metals," Philos. Mag. 43, 153 (1952).

${ }^{5}$ L. Dobrzynski, "Interface response theory of composite systems," Surf. Sci. 200, 435 (1988)

${ }^{6}$ L. Dobrzynski, "Interface response theory of discrete superlattices," Prog. Surf. Sci. 26, 103 (1987).

${ }^{7}$ A. Akjouj, B. Sylla, and L. Dobrzinski, "Introduction to a theory of composite systems-Simple examples of lamellar materials," Ann. Phys. 18, 363 (1993).

${ }^{8}$ H.-W. Lee, "Generic transmission zeros and in-phase resonances in timereversal symmetric single channel transport," Phys. Rev. Lett. 82, 2358 (1999).

${ }^{9}$ A. Yacoby, M. Heiblum, D. Mahalu, and H. Shtrikman, "Coherence and phase sensitive measurements in a quantum dot," Phys. Rev. Lett. 74, 4047 (1995)

${ }^{10} \mathrm{~T}$. Taniguchi and T. Buttuker, "Friedel phases and phases of transmission amplitudes in quantum scattering systems," Phys. Rev. B 60, 13814 (1999).

${ }^{11}$ P. Zanardi, "Virtual quantum systems," Phys. Rev. Lett. 87, 077901 (2001).

${ }^{12}$ M. A. Nielsen and I. L. Chuang, Quantum Computation and Quantum Information (Cambridge University Press, Cambridge, 2000).

${ }^{13}$ S. W. Hla, K.-F. Braun, B. Wassermann, and K.-H. Rieder, "Controlled low-temperature molecular manipulation of sexiphenyl molecules on $\mathrm{Ag}(111)$ using scanning tunneling microscopy," Phys. Rev. Lett. 93, 208302 (2004).

${ }^{14} \mathrm{P}$. Cellia and S. Gonella, "Manipulating waves with LEGO® bricks: A versatile experimental platform for metamaterials architectures," Appl. Phys. Lett. 107, 081901 (2015). 